\title{
Acquiring Variable Moment Arms for Index Finger Using a Robotic Testbed
}

\author{
Ashish D. Deshpande*, Ravi Balasubramanian, Jonathan Ko, and Yoky Matsuoka
}

\begin{abstract}
Human level of dexterity has not been duplicated in a robotic form to date. Dexterity is achieved in part due to the biomechanical structure of the human body and in part due to the neural control of movement. We have developed an anatomically correct testbed (ACT) hand to investigate the importance and behavioral consequences of anatomical features and neural control strategies of the human hand. One of the critical aspects of understanding dexterity is the analysis of the relationships between the hand muscle movements and joint movements, defined by the moment arms of the muscles. It is known that the moment arms for the hand muscles are configuration-dependent and vary substantially with change in posture. This paper presents a methodology for determining continuous variations in the moment arms with respect to multiple joints moving simultaneously. To determine variations in the moment arms of the ACT hand index finger muscles, we employed a nonparametric regression method called Gaussian processes (GPs). GPs give a functional mapping between the joint angles and muscle excursions, and the gradients of these mappings are the muscle moment arms. We compared the moment arm relationships of the ACT hand with those determined from the available cadaver data. We present the implications of the determination of variable moment arms toward understanding of the biomechanical properties of the human hand and for the neuromuscular control for the ACT hand index finger movements.
\end{abstract}

Index Terms-Index finger, moment arms, robot hands.

\section{INTRODUCTION}

$\mathbf{H}$ UMAN hands are capable of many dexterous grasping and manipulation tasks. Hand dexterity can be defined as the ability to precisely control movements and forces of all the DOFs of the hand to achieve a variety of tasks. Some of the examples of the human hand dexterity are the ability to play musical instruments with our hands, use chopsticks, gesture, and perform daily tasks, such as cooking and writing. Numerous anthropomorphic robotic hands have been developed with the goal to replicate such human-level dexterity [7], [9], [13], [22], [31]. Most of the existing and emerging robotic hands [21], [32] are developed as prosthetic devices, and hence, the focus has been

Manuscript received October 15, 2009; revised January 15, 2010 and March 9, 2010; accepted March 9, 2010. Date of publication May 3, 2010; date of current version July 14, 2010. Asterisk indicates corresponding author.

${ }^{*}$ A. D. Deshpande is with the Department of Mechanical Engineering, University of Maine, Bangor, ME 04469 USA (e-mail: ashish.deshpande@ maine.edu).

R. Balasubramanian is with the Department of Mechanical Engineering, Yale University, New Haven, CT 06520-8283 USA.

J. Ko and Y. Matsuoka are with the Department of Computer Science and Engineering, University of Washington, Seattle, WA 98105 USA.

Color versions of one or more of the figures in this paper are available online at http://ieeexplore.ieee.org.

Digital Object Identifier 10.1109/TBME.2010.2048326 on making them lightweight and easy to power and operate. We have developed a hand called the anatomically correct testbed (ACT) hand to mimic and study the biomechanics and neuromuscular controls of the human hand. We believe that understanding of human biomechanical structure and neural coding of the hand movements gained by experimenting with the ACT hand will lead to future developments of the robotic and prosthetic hands. This paper presents a technique to quantify the relationships between the muscle contractions and joint movements in human hands by studying these properties in the ACT hand.

An important characteristic of the human hand is the mechanical advantage, or the moment arm, that each muscle-tendon combination has on each joint. The relationship between the muscle-tendon excursions and joint movements, which is characterized by the moment arms, depends on the bone shapes over which tendons travel and the structure of the tendon network. In case of the index finger, a moment arm matrix can be defined to relate excursions of seven muscles to four independent joint motions. From cadaveric and human studies, it is known that the moment arms for the hand muscles are nonlinear function of all the joint angles [2], [10], [18]. Since the moment arm matrix relates the muscle excursions to the joint motions and also the muscle forces to the joint torques, understanding the variations of the moment arm matrix is critical for understanding the hand control strategies and hand dexterity.

In hand biomechanics research, the importance of variable moment arms of the finger muscles in achieving finger functionality, especially, for the index finger, has long been discussed. Brand et al. [10] collected excursion data and determined the moment arms for one of the index finger muscles in intact and excised cadaver hands, in order to study how pulley advancement affects moment arms and resulting changes in finger flexion and grip strength. An et al. [2] studied muscle excursions as functions of all four index finger angles in cadaver hands and determined the moment arms in order to study the effects of tendon transfer procedures. Recently, Kamper et al. [23] have studied the mapping between muscle activation and isometric joint torque production in the index finger of healthy subjects. The study concluded that the mapping was profoundly affected by finger posture and the variations in the torques were produced predominantly by changes in the moment arms. Buford et al. [12] have demonstrated that the moment arms need to be determined in order to calculate the exact contributions of intrinsic muscles to the finger joint moment. Weiss et al. [42] have studied the effectiveness of hand implants to replace metacarpophalangeal (MCP) joint by studying variations in the instantaneous center of rotation (COR) and moment arms after 


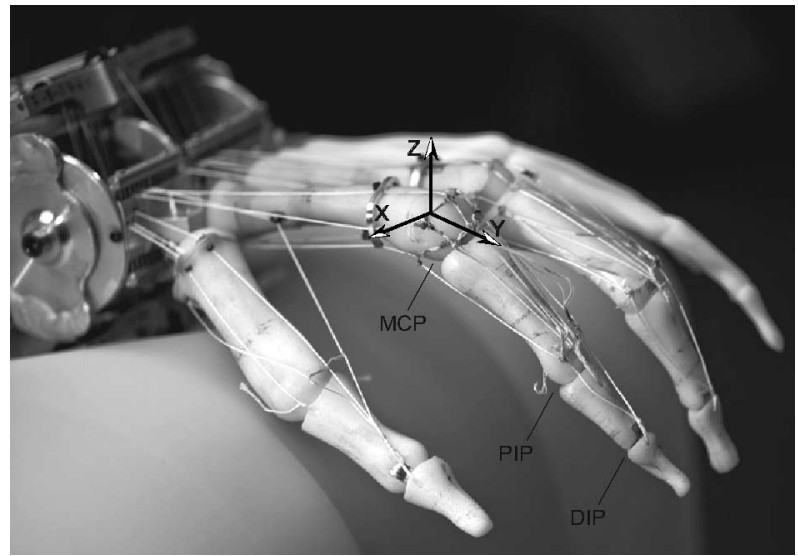

Fig. 1. ACT hand. The ACT hand is modeled to be human size. The skeletal structure and tendon routing in each finger are anatomical (Photo by Ellen Garrens).

MCP joint replacements with three different types of implants. Instantaneous and variable moment arms have also been studied for other joints, for example, for elbow [3], [17] and shoulder [27] joints. However, techniques used so far cannot be applied to determine continuously varying moment arms for hand muscles as multiple joints move simultaneously.

Although it has been accepted that the moment arms for muscles in the fingers are posture-dependent, the exact mappings of moment arms and finger postures have been difficult to determine, since it requires simultaneous measurement of all joint angle variations and muscle excursions. To address this, researchers have determined moment arms with respect to only limited and discrete finger motions. Due to the significance of MCP flexion for the entire finger movement, many studies have focused on the determination of moment arms for the index finger muscles with respect to only MCP flexion [10], [12], [42]. An et al. [2] present a comprehensive study on index finger moment-arms determination in cadaver hands by moving one finger joint at a time while fixing the other three joints. Fowler et al. [18] determine the moment arms in cadaver hands using MRI scans to measure the distances between tendons and joints in different finger postures. However, in this study, the moment arms are determined only at five finger postures, and the effects of tendon sliding, important in causing moment arm variations, are not captured. Exactly how the human moment arms vary with simultaneous joint movements in the human hand and how (or whether) the central nervous system (CNS) utilizes the variable moment arms to achieve the desired movement is not fully understood.

We attempt to answer these questions using the ACT hand shown in Fig. 1. The local nonlinear interactions between the muscle excursions and joint movements are mimicked in the $\mathrm{ACT}$ hand by bone shapes that match human bones and by a structure of tendons that connect the actuators to the finger bones. When a robotic system imitates the human structure, as in the ACT hand, identifying robotic hardware properties results in uncovering human biomechanical properties that might be challenging to identify in vivo. The goals of this paper are to

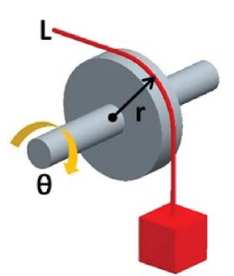

(a)

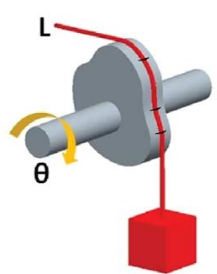

(b)

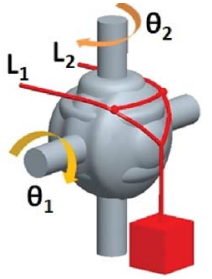

(c)
Fig. 2. Examples of moment arm variations with varying degree of complexity in the relation between angle and string slide length. (a) Moment $\operatorname{arm} R$ is simply $r$. (b) Moment arm is configuration-dependent and is given by: $R(\theta)=\partial L / \partial \theta$ (c) Moment arm matrix is dependent on two angles and is given by: $R_{2 \times 2}$ with elements $R_{i j}=\partial L_{i} / \partial \theta_{j}, i, j=1,2$.

present a system-identification method that allows, for the first time, the determination of the moment arm matrix for index finger muscles as a function of multiple joints moving simultaneously, and to discuss the implications of the variable moment arms.

Determination of the moment arm properties requires data collection over many trials, which is difficult to achieve with the cadaver fingers because the cadaver samples can only be used for limited time period and measurement of muscle displacements is difficult. Using the ACT hand, we have collected a large amount of data for finger motions. We implement, for the first time, a method called Gaussian processes (GPs) to determine the moment-arm functional mappings in a high-dimensional space (six muscles and four joints) with a large dataset. GP has advantages with high-dimensional data, since it is completely data-driven and can model highly nonlinear functions. We compare the moment arm relationships with those determined from the available cadaver data. We discuss the implications of the determination of variable moment arm matrix toward the understanding of the biomechanical properties of the human hand and for the neuromuscular control for the ACT hand index finger movements. Preliminary results on this topic are presented in [14].

\section{ACT INDEX FINGER DESCRIPTION}

In this section, we present important features of the index finger of the ACT Hand, which influence the moment arm determination. The details of finger skeletal structure and the tendon routing have been presented earlier in [41] and [43].

\section{A. Finger Structure and Muscles}

Fig. 1 shows the ACT index finger we used for the systemidentification experiment. The finger consists of four bones (one metacarpal and three phalanges), which are connected by three joints, namely, MCP, proximal interphalangeal (PIP), and distal interphalangeal (DIP). The finger has 4 DOF, abductionadduction (Ab-Ad) at MCP and flexion-extension at all joints. The finger is controlled by three intrinsic muscles, namely, palmar interossei (PI), radial interossei (RI), and lumbrical (LUM), and three extrinsic muscles, namely, extensor interossei (EI), flexor digitorum superficialis (FDS), and flexor digitorum 
profundus (FDP). The human index finger has additional extrinsic muscle called extensor digitorum communis (EDC). This muscle is not copied in the ACT hand, since it is equivalent to EI when the movements of a single digit are analyzed. The bone shapes and an extensor hood mechanism, which is a complex web of extensor tendons located on the dorsal side of the finger, are duplicated to match the human counterpart [43].

Each muscle is realized by a brushless dc motor and the tendons are connected to the motor shafts. In the human hand, the LUM muscle does not attach to a bone, instead it attaches proximally to the tendons of flexor digitorum profundus and distally to extensor expansions on the dorsal surface of the hand. This arrangement is not realized in the ACT hand due to its complexity. Instead, the LUM tendon and associated motor are attached to the equivalent of a skeletal anchor point. We hope to achieve anatomical LUM muscle behavior with software control of the motors. A constant-torque spring is installed on each motor shaft, which generates pretensions $(0.3 \mathrm{~N})$ in the tendons to avoid tendon slacking. Each motor is connected to a miniature controller with an embedded photosensor and an encoder wheel with 114 ticks/degree, allowing for a high-precision position sensing of the motor rotation [5]. The controllers are connected to a real-time application interface (RTAI) Linux [38] machine that provides motor position readings at a high frequency $(500 \mathrm{~Hz})$.

\section{B. Moment Arm Variations}

The moment arm relates the rate of change of finger joint angles to the rate of change of muscle excursions. A positive moment arm means that the muscle length increases when the joint angle increases, while a negative moment arm means that the muscle length decreases as the joint angle increases. In the case of the ACT hand index finger, the moment arm is defined by a matrix $R$ of dimension $6 \times 4$. The elements of this matrix can be determined by first determining the functional mappings between the joint and muscle movements. The finger DOFs and the muscle excursions can be defined to be related by functions $f_{i}$ as follows:

$$
l_{i}=f_{i}(\underline{\theta}), \quad i=1, \ldots, 6
$$

where $l_{i}$ is the excursion length for the muscle $i$, which is a member of the vector of muscle excursions $\left(\underline{l}=\left[l_{1}, l_{2}, l_{3}, l_{4}, l_{5}, l_{6}\right]^{T}\right)$ and $\underline{\theta}$ is a vector of finger joint angles $\left(\underline{\theta}=\left[\theta_{1}, \theta_{2}, \theta_{3}, \theta_{4}\right]^{T}\right)$. We define $\theta_{1}$-MCP Ab-Ad, $\theta_{2}$ - MCP Flex, $\theta_{3}$ - PIP Flex and $\theta_{4}$-DIP Flex, with the abduction and flexion as positive directions. The moment arm matrix relates the joint angular velocities to the muscle length rate of changes as follows:

$$
\underline{i}=R(\underline{\theta}) \underline{\dot{\theta}}
$$

where

$$
R_{i j}(\underline{\theta})=\frac{\partial l_{i}}{\partial \theta_{j}}=\frac{\partial f_{i}}{\partial \theta_{j}}, \quad i=1, \ldots, 6 \text { and } j=1, \ldots, 4 .
$$

The moment arm matrix also defines the relationship between the muscle forces and joint torques as follows:

$$
\underline{\tau}=-R(\underline{\theta})^{T} \underline{f}_{m}
$$

where $\underline{\tau} \in \mathbb{R}^{4 \times 1}$ is a vector of joint torques and $\underline{f}_{m} \in \mathbb{R}^{6 \times 1}$ is a vector of muscle pull forces.

\section{Method FOR Data Collection AND ANAlysis}

To determine the moment arms, the index finger of the ACT hand was moved in the entire range of its joints. The operator held the finger tip and systematically moved the finger to the extreme position of each joint. Based on this data, a visual 4-D map of the entire finger workspace was generated using the $\mathrm{C}++$ programming and OpenGL platform. The operator then moved the finger to all the possible positions in its workspace multiple times with the aid of the data visualization tool.

The muscle excursion data were collected by measuring the angular rotations of motors using encoders on the motor shaft. During the movements, the motors did not apply any torque. The joint angle data were collected using a motion capture system called Vicon 360. Six Vicon cameras of the type M2 were set up around the finger to record motions involving all four joint angles. Thirteen markers, each $3 \mathrm{~mm}$ in diameter were placed on the ACT finger and the distribution of the markers was as follows: five on the MCP bone, three on the proximal and middle phalange, and two on the distal phalange. The locations of the markers on the bones were chosen to avoid marker occlusion during the finger movements. The $X Y Z$ positions of the markers were recorded at $120 \mathrm{~Hz}$, and finger joint angles were determined by using an angle determination algorithm built into the Vicon software (Vicon iQ 2.5). Each data point for our experiments consisted of the time stamp, the muscle positions, and the joint angles. We collected approximately 200000 data points that cover the whole range of four joint angles, which is: MCP Ab-Ad: $\in\left[-15^{\circ} 15^{\circ}\right]$, MCP Flex-Extend: $\in\left[-30^{\circ} 90^{\circ}\right]$, PIP Flex-Extend: $\in\left[0^{\circ} 110^{\circ}\right]$, and DIP Flex-Extend: $\in\left[0^{\circ} 70^{\circ}\right]$. On average, for every 4-D bin defined by $5^{\circ}$ interval in the joint angles, we have 820 (SD 98.1) data points, which shows a satisfactory coverage of the data in the finger workspace.

The determination of the functional mapping, given by (1) in a high-dimensional space (six muscles and four joints) with a large dataset, is challenging. To address this, we implemented a machine-learning technique called the GP. A GP-based regression model is a probabilistic kernel method [16], [26]. GP has advantages with high-dimensional data, since it is completely datadriven and can model highly nonlinear functions. This method is explained in detail in the Appendix. By taking the derivative of the joint angle to muscle length mappings we can determine the moment arms. GP has a unique property that it allows for the determination of the gradient of the nonparametric functional mapping given by GP. For details on GP gradients, see [37].

\section{RESULTS AND VALIDATION}

\section{A. Muscle Excursions}

Table I shows the total excursion lengths for all muscles for the full range of joint angle movements. Table II shows muscle excursion lengths for $100^{\circ}$ change in joint angle for both the ACT hand and cadaver hands [2] (see shortly for more on comparisons with cadaver data). 
TABLE I

MAXimum Muscle EXCursions (IN Millimeters) OBSERVEd OVER AlL COMBINATIONS OF JOINT ANGLES

\begin{tabular}{|l|c|c|c|c|c|c|}
\hline & EI & PI & FDP & LUM & FDS & RI \\
\hline$l_{\max }$ & 28.56 & 14.17 & 36.12 & 18.87 & 30.78 & 29.44 \\
\hline
\end{tabular}

TABLE II

Muscle EXCURSIONS (IN Millimeters) FOR $100^{\circ}$ OF ANGLE CHANGE

\begin{tabular}{|c|c|c|c|c|c|c|}
\hline & EI & PI & FDP & LUM & FDS & RI \\
\hline$\theta_{1} \mathrm{ACT}$ & 1.82 & 15.99 & 0.89 & 16.14 & 0.33 & 17.96 \\
\hline Cad. & 3.48 & 10.17 & 2.92 & 8.10 & 3.99 & 11.46 \\
\hline$\theta_{2} \mathrm{ACT}$ & 20.31 & 12.15 & 18.80 & 0.10 & 18.92 & 13.13 \\
\hline Cad. & 15.07 & 7.87 & 18.98 & 15.26 & 21.04 & 5.88 \\
\hline$\theta_{3} \mathrm{ACT}$ & 4.98 & 0.21 & 11.82 & 3.00 & 11.68 & 0.32 \\
\hline Cad. & - & - & - & - & - & - \\
\hline$\theta_{4}$ ACT & 0.46 & 1.46 & 3.83 & 2.67 & 0.71 & 7.79 \\
\hline Cad. & - & - & - & - & - & - \\
\hline
\end{tabular}

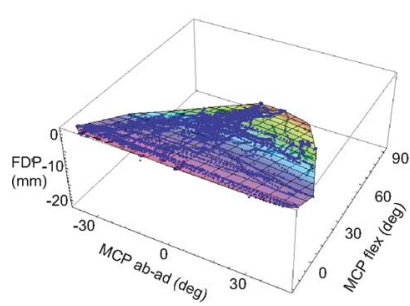

(a)

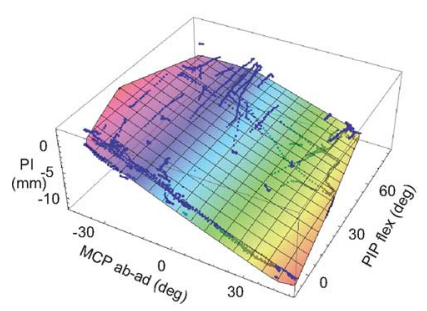

(c)

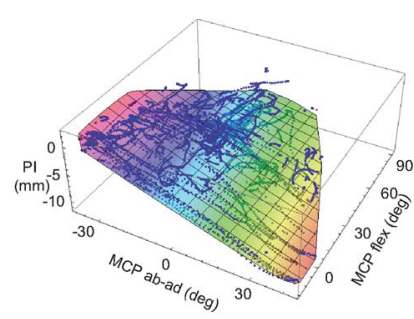

(b)

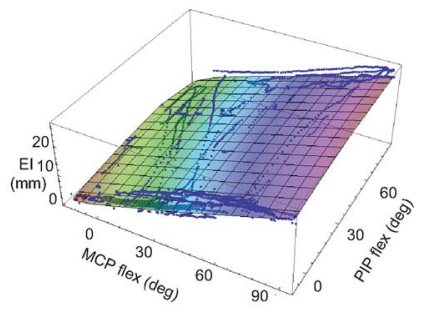

(d)
Fig. 3. FDP, PI, and EI muscle excursions (in millimeters) as functions of two finger angles, while other two angles are held constant at $0^{\circ}$ for the sake of plotting. The surfaces were fitted using the finger motion data shown in dots. (a) FDP excursions. (b) PI excursions. (c) PI excursions. (d) EI excursions.

Fig. 3 shows example plots of muscle excursions, for FDP, PI, and EI muscles, as functions of two joint angles, while the other two joint angles are held constant at $0^{\circ}$ for the sake of plotting. The mean error across all data points with the GP-based mapping is 0.65 (SD 0.33) $\mathrm{mm}$. This error is quite low when compared with the total excursions, as given in Table II, which gives the muscle excursions per $100^{\circ}$ of angle change. Thus, we have a functional mapping between the joint angles and muscle excursion. As expected, the extrinsic muscles (FDS, FDP, and EI) show large excursions with finger flexion and smaller excursions for abduction-adduction; while the intrinsic muscles (LUM, PI, and RI) show large excursions with finger abduction-adduction and smaller excursions for flexion. GPs provide a measure of uncertainty for each data point along with mean estimation. Table III gives the mean uncertainty for each muscle.
TABLE III

MEAN UNCERTAINTY $u_{m}$ AND STANDARD DEVIATION FROM THE MEAN UNCERTAINTY (IN MILLIMETERS) GIVEN BY GP-BASED MAPPING OF MUSCLE EXCURSIONS FOR EACH MUSCLE

\begin{tabular}{|c|c|c|c|c|c|c|}
\hline & EI & PI & FDP & LUM & FDS & RI \\
\hline$u_{m}$ & 1.20 & 0.90 & 0.32 & 1.50 & 0.11 & 0.83 \\
std & $(0.12)$ & $(0.19)$ & $(0.03)$ & $(0.07)$ & $(0.02)$ & $(0.01)$ \\
\hline
\end{tabular}

\section{B. Moment Arms}

Using the aforementioned functional relationship between the joint angles and muscle excursions, we computed moment arms using (3). Table IV gives the mean, maximum, and minimum values (in millimeters) of the moment arm for all six muscles. The moment arms for the muscles with respect to all four joint angles show substantial variations. The moment arms of some of the muscles with respect to the MCP angles are different from the results in [14], where we held the PIP and DIP angles constant during the experiments. This shows that the moment arms with respect to the MCP joint angles are affected by the changes in the PIP and DIP angles. Fig. 4 gives the variations in the moment arms for the muscles FDP, EI, and PI with respect to two joint angles, while the other two joint angles are held constant at $0^{\circ}$ for the sake of plotting. We notice that the large variations in the FDP's moment arm with respect to MCP flexion is primarily a function of DIP and MCP Ab-Ad configuration. However, EI's moment arm with respect to PIP varies significantly with PIP flexion. The 2-D projections of PI's moment arms show that PI's moment arms do not vary much in the MCP Ab-Ad and PIP joint angle space. Fig. 5 shows the variations of moment arms estimations for all muscles when PIP and DIP joints are varied individually while other finger joints are held constant. The shaded regions show the uncertainty or variance given by GPs.

\section{Cadaver Data Comparison}

We compare our results with index finger cadaver data from [2], which is one of the most comprehensive datasets available. Data in [2] is from independent variations in each joint while other joints are fixed. Table II shows the muscle excursions with $100^{\circ}$ of joint changes for a cadaver hand and the ACT hand. We have regenerated the moment arm variations from [2] (from one female specimen) in Fig. 6(b) and (d). To make the comparison between variable moment arms data, we generated slice plots from our data, as shown in Fig. 6(a) and (c). We generated these plots by keeping the other joint angles constant as it was done for the cadaver data. The signs of moment arms for all the muscles match, except for LUM in parts of finger flexion, confirming that the elongation and contraction of all the muscles are copied in the ACT hand. Note that the sign of MCP Ab-Ad angle is switched to match with the cadaver data leading to change in the sign of the moment arms in Fig. 6(a). Table V gives the correlation coefficients between individual muscle plots for MCP Ab-Ad and flexion variations. The differences in variations in LUM and RI with flexion angle and PI with adduction might have arisen due to the differences in the structure of LUM in the cadaver hands and the ACT hand. 
TABLE IV

Moment Arm (in Millimeters per Radians) Mean, MaX, And Min for All SiX Muscles of the ACT Hand Due to Changes in Finger Angles

\begin{tabular}{|c|c|c|c|c|c|c|c|c|c|c|c|c|}
\hline & \multicolumn{3}{|c|}{$\begin{array}{l}\text { MCP } \\
\text { Ab-Ad }\end{array}$} & \multicolumn{3}{|c|}{$\begin{array}{l}\text { MCP } \\
\text { Flex- } \\
\text { Extend }\end{array}$} & \multicolumn{3}{|c|}{$\begin{array}{l}\text { PIP } \\
\text { Flex- } \\
\text { Extend }\end{array}$} & \multicolumn{3}{|c|}{$\begin{array}{l}\text { DIP } \\
\text { Flex- } \\
\text { Extend }\end{array}$} \\
\hline & mean & $\max$ & $\min$ & mean & $\max$ & $\min$ & mean & $\max$ & $\min$ & mean & $\max$ & $\min$ \\
\hline EI & 0.11 & 9.35 & -13.97 & 12.27 & 25.70 & 8.68 & 3.25 & 8.56 & -1.78 & -0.20 & 1.89 & -4.07 \\
\hline PI & -8.24 & -1.50 & -13.00 & -0.90 & 3.95 & -6.04 & 0.11 & 3.32 & -2.61 & 1.12 & 3.26 & -1.23 \\
\hline FDP & -0.46 & 7.60 & -8.83 & -10.82 & -9.39 & -15.76 & -6.35 & -3.32 & -8.99 & 4.40 & 3.32 & -6.89 \\
\hline LUM & 8.31 & 14.75 & 0.77 & 0.26 & 10.27 & -9.01 & 1.26 & 10.21 & -5.97 & $\mathbf{0 . 0 3}$ & 2.51 & -3.14 \\
\hline FDS & 0.10 & 8.07 & -13.74 & -11.26 & -9.23 & -15.77 & -6.35 & -4.25 & -8.33 & 0.03 & 1.31 & -1.58 \\
\hline RI & 8.42 & 16.31 & -7.94 & -5.95 & 5.39 & -21.57 & -0.11 & 10.93 & -6.48 & 3.74 & 6.33 & 1.36 \\
\hline
\end{tabular}

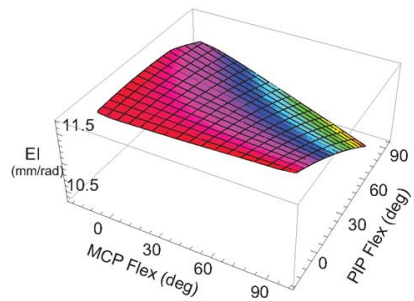

(a)

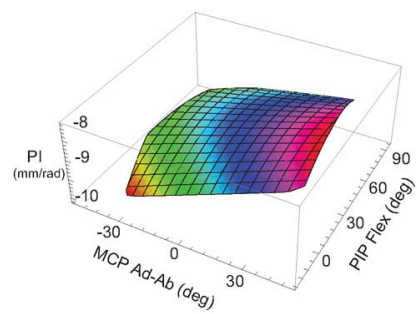

(c)

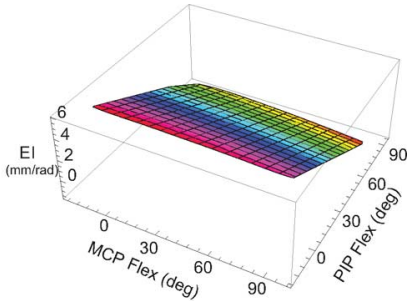

(b)

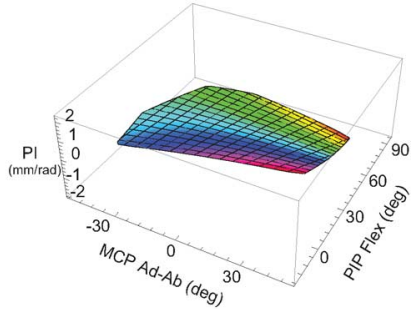

(d)

Fig. 4. Moment arm variations for EI and PI muscles as two finger angles vary. The other two finger angles are held constant at zero for the sake of plotting. (a) and (b) show the variations of the moment arms of the muscle EI $\left(l_{1}\right)$ with respect to two separate angles, MCP flex $\left(\theta_{2}\right)$ and PIP flex $\left(\theta_{3}\right)$ are shown. Therefore, (a) gives the variations of $\partial l_{1} \partial \theta_{2}$ and (b) gives the variations of $\partial l_{1} \partial \theta_{3}$. (a) EI moment arm w.r.t. MCP flex. (b) EI moment arm w.r.t. PIP flex. (c) PI moment arm w.r.t. MCP Ab-Ad. (d) PI moment arm w.r.t. PIP flex.

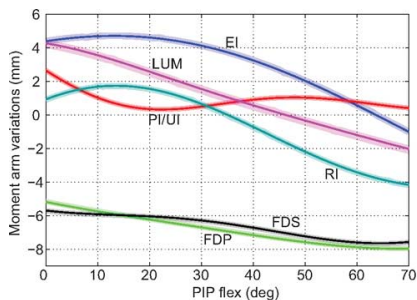

(a)

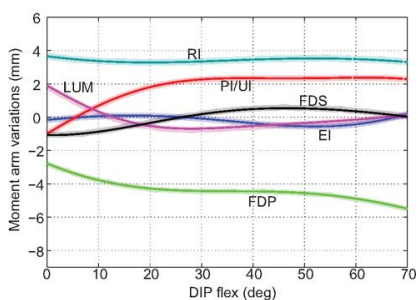

(b)

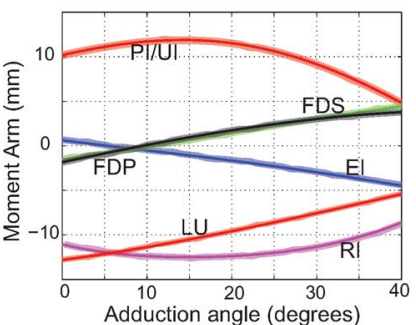

(a)

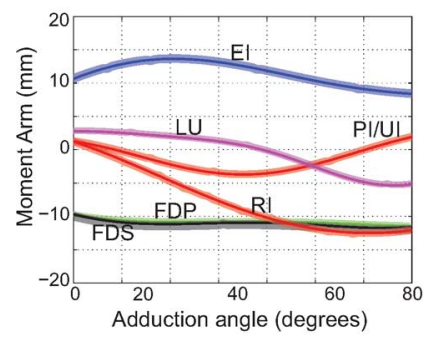

(c)

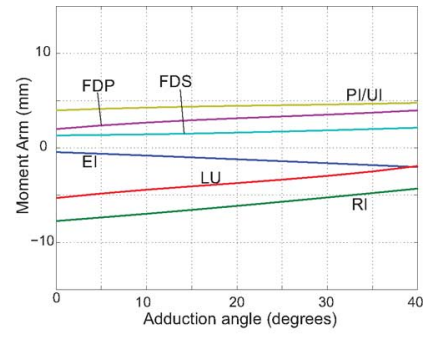

(b)

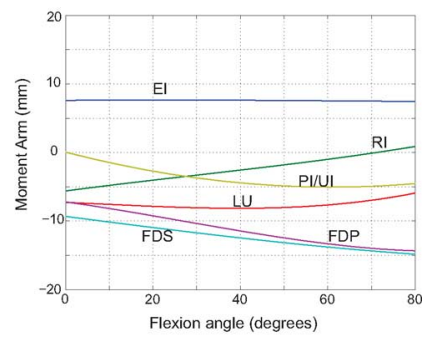

(d)

Fig. 6. Variations of moment arm for the six index finger muscles as MCP joint angles vary, while PIP and DIP angles are fixed at $0^{\circ}$ for the sake of plotting. The shaded regions in (a) and (c) show the uncertainty or variance given by GPs. (b) and (d) Recreated using the data and figures provided in [2]. The sign of the moment arms, indicating contribution to flexion/extension or abduction/adduction, for all muscles match, except for LUM in parts of finger flexion, with the cadaver plots. The moment arm values are higher in our case by, on average by $25 \%$, presumably, at least in part, due to the fact that we modeled the ACT hand size on the size of a male subject and data in An et al. [2] is from a female specimen. (a) Ad-Ab ACT. (b) $\mathrm{Ad}-\mathrm{Ab}$ Cadaver. (c) MCP Flex ACT. (d) MCP Flex Cadaver.

TABLE V

CORRELATION COEFFICIENTS BETWEEN MOMENT ARM CURVES FROM THE CADAVER AND ACT HAND GIVEN BY Fig. 6

\begin{tabular}{|c|c|c|c|c|c|c|}
\hline & EI & PI & FDP & LUM & FDS & RI \\
\hline MCP FE & 0.94 & 0.47 & 0.83 & -0.71 & 0.85 & -0.95 \\
MCP AA & 0.99 & -0.66 & 0.99 & 0.67 & 0.93 & 0.99 \\
\hline
\end{tabular}

Fig. 5. Variations of moment arms for the six index finger muscles as PIP and DIP joint angles vary, while $\mathrm{MCP}$ angles are fixed at $0^{\circ}$, for the sake of plotting. The shaded regions show the uncertainty or variance given by the GP. Since the variability is very small, the shading is hard to see. The figures indicate that variations in the moment arms due to changes in PIP and DIP angles are considerable and must be accounted for in the moment arm structure. (a) PIP effects on R. (b) DIP effects on R.

\section{Validation}

To validate the moment arm matrix estimations, we moved the ACT index finger in a test path and collected muscle excursion and joint angle data. We then used the moment arm matrix $R(\theta)$ 
determined by using GP to predict finger joint angles given muscle excursions, using the relationship

$$
\tilde{\theta}=\int R(\underline{\theta})^{-1} i d t
$$

The fingertip position was calculated using the joint angles determined by using (5) and also using the joint angles collected during the test.

Currently, in many hand biomechanics studies, moment arms for the index muscles are assumed to be constant due to lack of available moment arm variation models. To demonstrate the effects of such an assumption, we predicted the finger path using a constant moment arm matrix $R_{c}$, given by (6). The constant moment arm values were determined by measuring the approximate distance between the joint centers and tendon locations from the ACT finger drawings when all the joint angles are at zero

$$
R_{c}=\left[\begin{array}{cccc}
0.10 & 9.80 & 5.08 & 3.16 \\
8.05 & 2.92 & 4.45 & 3.16 \\
2.97 & 6.43 & -5.08 & -3.81 \\
5.87 & 5.54 & 4.45 & 0 \\
1.68 & 5.59 & -5.08 & 0 \\
9.35 & 1.42 & 2.54 & 3.16
\end{array}\right] \mathrm{mm} / \mathrm{rad} .
$$

Fig. 7 shows the comparison of the paths estimated by using GP, by using the constant moment arms assumption, and the true fingertip path determined by using the recorded joint angles. The shaded regions in the 2-D projections show the finger tip position calculated by using the variance in joint angles given by GP. Table VI gives the $r^{2}$ value between the ground truth and the estimated paths, and also the mean error. The mean error was calculated as the $L_{2}$-norm distance between the true path and the estimated path in 3-D.

\section{DISCUSSION}

In this paper, we present a method, for the first time, for the investigation of the variations in the index finger moment arms when multiple joints move simultaneously. The determination of a mathematical model of variations in the moment arms for the index finger muscles is the main contribution of this paper. In this section, we discuss our results and also present the implications of our findings.

\section{A. Comparison with Cadaver}

The determination of the variable moment arms using cadavers is difficult due to the challenges of preparations required for the cadaver experiments, the limited availability of specimen, and limited time for experiments and difficulty of experimental setup with the thawed specimen. With the ACT hand, we are able to collect extensive data (over 200000 data points), which allows us to determine exact mapping between motions of all the muscles and all finger angles. Of course, the method of using data from a robotic structure to infer human properties has limitations. Although we have mimicked the human biomechanics, some differences exist. For example, the ACT hand does not possess the musculotendon viscoelastic properties. Also, the differences in the structure of LUM in the cadaver hands and

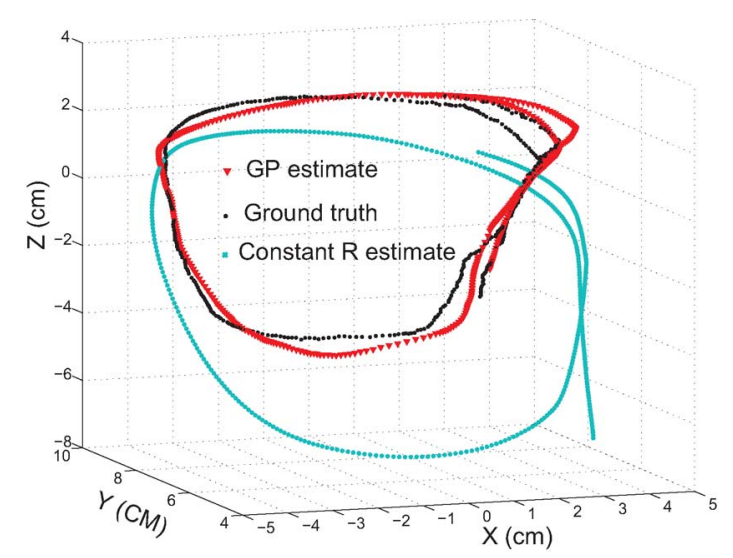

(a)

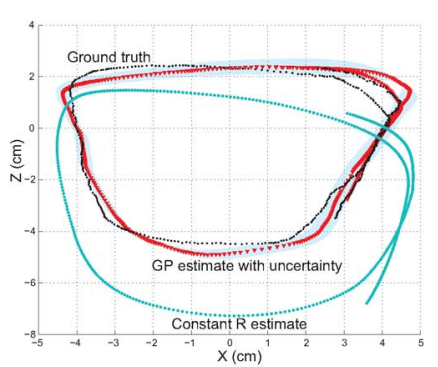

(b)

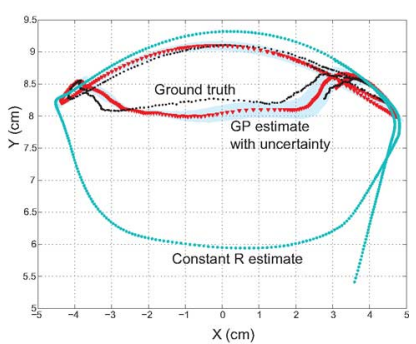

(c)
Fig. 7. Validation of moment arms. The 3-D path is shown in (a). (b) and (c) 2-D projections of the same path. The shaded regions in the 2-D projections show the finger tip position based on the variance in joint angles given by GP. Figures show the ground truth trajectory and the trajectories estimated by using the moment arms from the GP method and a constant moment arm given by (6). (a) Validation 3-D. (b) $X Z$ projection. (c) $X Y$ projection.

TABLE VI

Validation Results: The Path Estimated Using GP Matches Closely $\left(r^{2}\right.$ Value $)$ To the Ground Truth Path

\begin{tabular}{|c|c|c|}
\hline Method & $r^{2}$ value & Mean error $(\mathrm{se})(\mathrm{mm})$ \\
\hline GP & 0.98 & $0.46(0.23)$ \\
Constant matrix & 0.67 & $3.58(1.27)$ \\
\hline
\end{tabular}

the ACT hand lead to differences in the variations in the moment arms. When controlling the finger, we have the ability to control individual muscle force and movements. To achieve anatomical LUM behavior, our plan is to couple the LUM muscle movements, using the software control algorithms, with those of the other hand muscles. The negative correlation (see Table V) between the RI muscle moment arms with respect to MCP flexion in the cadaver and ACT hand suggests that while the line of action of RI muscle moves away from the COR as MCP flexes in the ACT hand, it moves closer to the COR in the cadaver. The signs of the moment arms match in both cases, indicating that there are only a slight difference in the biomechanical structure of the ACT hand and cadaver specimen. Although PI muscle moment arms with respect to MCP Ab-Ad in the ACT hand and cadaver are negatively correlated (see Table V), both curves are fairly flat; therefore, negative correlation is acceptable. Overall, our results show that the ACT hand moment arms 
are comparable to the available cadaver results and the meaningful understanding of human moment arm variations can be achieved from the ACT hand experiments.

\section{B. Muscle Excursions and Variations in Moment Arms}

Our results demonstrate that the excursion lengths and moment arms for all muscles of the index finger depend significantly on all the joint positions of the finger. As observed in An et al. [2], the extrinsic muscle excursions show significant dependency on flexion, but little variation with abductionadduction. The intrinsic muscle excursions show significant dependency on both flexion and abduction.

Table IV shows that the moment arms for all muscles of the ACT hand index finger depend significantly on all the joint positions of the finger, and for some of the muscles, the moment arms change sign. This allows for the understanding of how the muscle switch roles between contributing and noncontributing to the joint motions. Such an understanding is critical for composing controllers for the finger motion and also for decoding brain control of the human muscles for achieving desired finger movements [4], [15]. Fig. 5 and comparisons with [14] indicate that the variations in the moments arms with respect to the PIP and DIP angles are considerable. Also, the moment arms for the extrinsic muscles of the ACT hand with respect to the flexion angles show large variations and maintain the sign, but show small variations and sign change with respect to the adduction angle. In contrast, the moment arms for the intrinsic muscles with respect to the flexion angles show small variations, but show large variation with respect to the abduction-adduction angle. Earlier studies to determine the contribution of intrinsic muscles in MCP flexion in the human hand did not consider variable moment arms [12], [29] and may need to be updated. Fig. 5 indicates that the variations in the moment arms due to changes in PIP and DIP angles are considerable and must be accounted for in the moment arm structure.

\section{Implications for Force and Velocity Control}

Determination of the variable moment arm matrix allows for the quantification of contributions of the muscle forces and velocities to the finger motions in various finger configurations.

Fig. 8 shows variations in the MCP flexion torque for various finger configurations when all six muscles are fully activated. This surface was created by using the relationship between the muscle forces and joint torques given by (4). Specifically

$$
\tau_{X}=\sum_{i=1}^{6}-\left(R^{T}(2, i) \times f_{m}(i)_{\max }\right)
$$

where $\tau_{X}$ is the flexion torque, $R$ is the variable moment arm matrix, and $f_{m}(i)_{\max }$ is the maximum pull force generated by muscle $i$. The maximum force values were used from the literature [34]. Fig. 8 illustrates that for the same forces applied by the muscles, the joint torques vary depending on the finger configurations. It shows that a constant moment arm, using (6), does not capture the variations in joint torques. With the variable moment arm matrix, the flexion torque is maximum when the

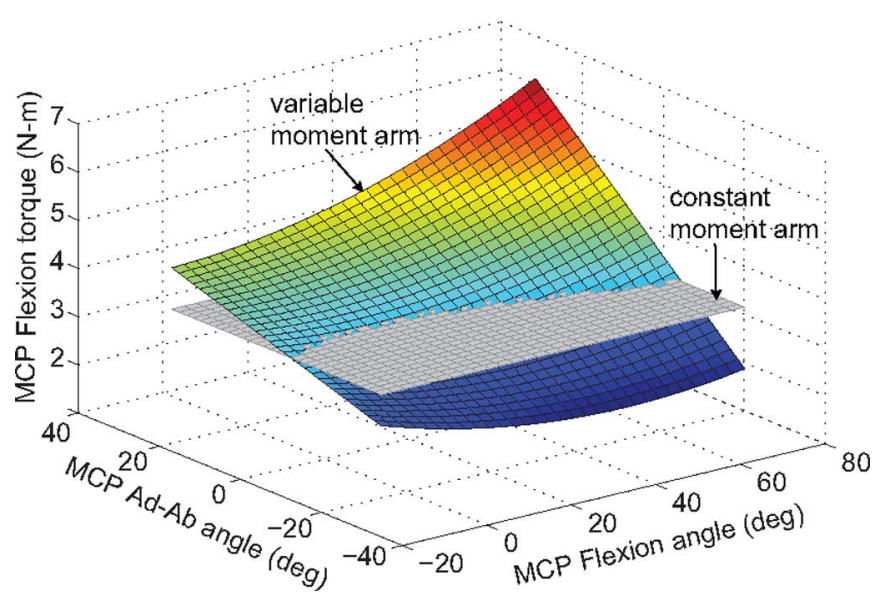

Fig. 8. MCP joint torque in the flexion $(X)$ direction for various finger configuration as all six muscles are fully activated. The flexion torque is maximum when the finger is adducted and flexed. The figures shows the variation with a variable moment arm and with a constant moment arm [see (6)].

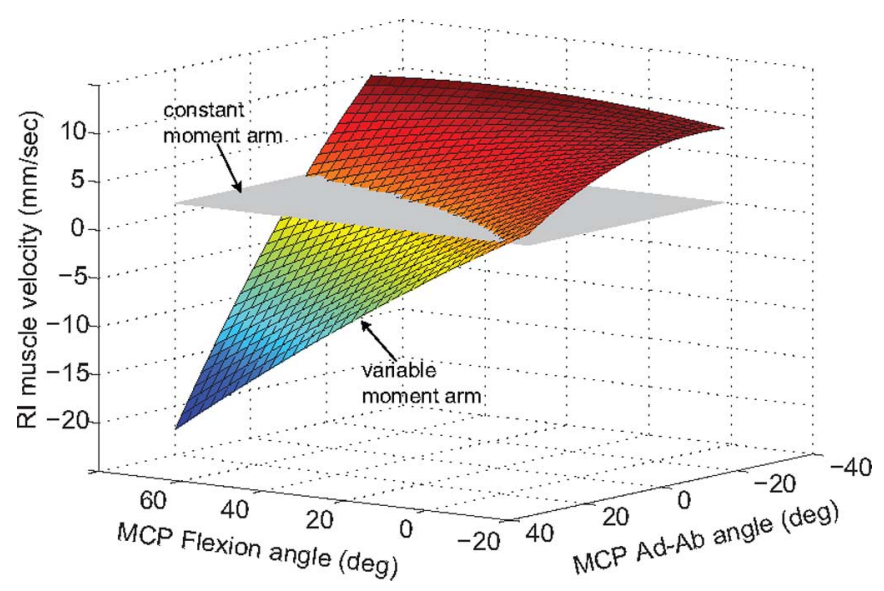

Fig. 9. Variations in the excursion velocity of the muscle RI for constant joint angle velocities. The figures shows the variation with a variable moment arm and with a constant moment arm [see (6)].

MCP joint is adducted and flexed. Physiologically, this allows the finger to produce the maximum palmar force when the finger is flexed and adducted. A study by Li et al. [30] in which the finger strength in various finger configurations was measured also concluded that the finger strength is highest when MCP joint is adducted and flexed. Our results have proven that variations in moment arms of the finger muscles might have played a significant role in achieving configuration-dependent variations in finger strength.

Understanding of variations in the moment arms is critical for the understanding of neuromuscular controls of the finger. For example, Fig. 9 shows a plot of variations in the instantaneous excursion velocity of the muscle RI for the same instantaneous angular velocities of MCP Ab-Ad (0.5 rad/s), MCP Flex $(1.0 \mathrm{rad} / \mathrm{s})$, PIP Flex $(1.0 \mathrm{rad} / \mathrm{s})$, and DIP Flex $(1.0 \mathrm{rad} / \mathrm{s})$, as the configurations of the MCP Ab-Ad and MCP Flex angles vary. As the MCP angles vary, while PIP and DIP Flex angles are kept constant, the RI velocity changes from positive to negative. This means that the muscle switched from being active and pulling 
to being passive and stretching. Fig. 9 also shows that a constant moment arm, using (6), does not capture the variations in muscle velocities as functions of the finger configuration. Thus, to control the finger joint velocities, the muscle velocities are generated based on the finger configuration using the variable moment arms. A constant moment arms assumption will lead to erroneous model of neuromuscular controls.

\section{Advantages of GPS}

To the best of our knowledge, this is the first time that a GP-based machine-learning technique is implemented to determine the moment arm variations. Our results show that a good mapping between angles and muscle-length excursions was derived using GP modeling. In the past, we have employed a least squares regression (LSR) method to this end [14]. LSR is parametric and simple to implement; however, the structure for the parametric equations need to be defined by the user and the quality of fit is dependent on the choice of structure. The GP-based modeling is parameter-less and the mappings are purely datadriven. GPs also provide estimated variance at each data point and it can be utilized in designing the controllers for the finger motion and force tracking [15]. One disadvantage of this approach is that it is computationally costlier than LSR [16], [26]. As a result, for example, one run of the control loop with GPbased mapping takes $28 \mathrm{~ms}$, whereas with LSR mapping takes $15 \mathrm{~ms}$. We have demonstrated that this is not a limitation in achieving fast finger control [15].

\section{E. Future Implications}

The investigation of the importance of variation in the moment arms in achieving the hand movement control may allow us to understand the neuromuscular control of human hands. By utilizing the variable moment arms, we have designed position and joint torque control strategies for the finger movements, as discussed in [15]. The determination of the variations in the moment arms is important for other hand applications as well. Determination of moment arm variations would help in testing the viability of hand implants [6], [40]. It would be an important part of a biomechanical model of finger dynamics [11], [28] and control [20]. So far, tendon arrangement and grasping abilities are analyzed with the assumption that the moment arms are constant [8], [19], [33], [34]. Our results will help to update these studies to model anatomical hand motions. Moment arm determination will also play an important role in developing the next generation of hand animations [1] and simulations [12], [39].

The knowledge of variations in joint torques and muscle velocities as functions of finger configurations is critical for understanding the neural control of the hand and for designing brain-machine interfaces for prosthetic hands. As shown in Fig. 8, with the variable moment arms, for given muscle forces, the joint torques vary significantly ( $>100 \%)$, while constant moment arms lead to constant joint torques. Therefore, a tight grasping task is easier to execute in the center of the hand than in other configurations. We believe that the CNS learns the models of local biomechanical properties, including variable moment arms and generates neural signals in accordance with biomechanical features. The current accepted theory of neuromuscular controls suggests that the CNS develops an internal model of the motor command given the states and tasks, changes in states given the motor command, and sensory feedback given the new state [24], [35], [44]. Researchers have concluded that internal models are fundamental for understanding a range of processes, such as state estimation, prediction, context estimation, control, and learning [44]. It may be possible that the CNS builds the internal models of the hand muscle moment arms to achieve dexterous control. Therefore, for the successful neural control of a prosthetic hand, we may have to mimic the anatomical features in the prostheses so that neural signal can be directly fed in. Otherwise, if the prostheses do not possess all the physiological features, then we may have to design software interfaces to decode and process the neural signals, using our models of the anatomical features, before sending those to the prostheses. On the other hand, considering the complexity of realizing anatomical features, it is worth exploring whether such anatomical details are necessary in the hand prostheses, joint implants, and computer graphics models. The variable moment arms are critical in neuromuscular control of the hand to achieve dexterity, and a constant moment arm assumption may lead to incorrect representation of the human hand. It is possible that in case of hand prostheses, detailed anatomical imitation may not be necessary and the brain can adapt to different hardware with limited features. In our opinion, the anatomical features will help in adaptation of brain control for prosthetic devices.

\section{APPENDIX}

\section{Gaussian Processes}

GPs are nonparametric techniques for learning regression functions from sample data [37]. GPs have been used with great success in robotics applications, such as reinforcement learning [16] and Bayesian filtering [25].

Assume that we have $n d$-dimensional input vectors $\mathbf{X}=\left[\mathbf{x}_{1}, \mathbf{x}_{2}, \ldots, \mathbf{x}_{n}\right]$ and associated scalar outputs $\mathbf{Y}=$ $\left[y_{1}, y_{2}, \ldots, y_{n}\right]$. The output $y$ is a function of $\mathbf{x}$ with added Gaussian noise

$$
y_{i}=f\left(\mathbf{x}_{i}\right)+\mathcal{N}\left(0, \sigma_{n}\right)
$$

where $\sigma_{n}^{2}$ is the variance of the zero-mean white noise.

A GP defines a zero-mean, Gaussian prior distribution over the function $f$. This function is inferred by querying at specific inputs a multivariate Gaussian conditioned on training data $X$ and $Y$. The Gaussian that related the inputs and outputs is in the form

$$
p(\mathbf{Y} \mid \mathbf{X})=\mathcal{N}\left(\mathbf{Y} ; 0, \mathbf{K}+\sigma_{n}^{2} \mathbf{I}\right)
$$

The covariance of this Gaussian distribution is defined via a kernel matrix $\mathbf{K}$ and a diagonal matrix with elements $\sigma_{n}^{2}$. The elements of the $n \times n$ kernel matrix $\mathbf{K}$ are specified by a kernel function over the input values: $\mathbf{K}[i, j]=k\left(\mathbf{x}_{i}, \mathbf{x}_{j}\right)$. By interpreting the kernel function as a distance measure, we see that if points $\mathbf{x}_{i}$ and $\mathbf{x}_{j}$ are close in the input space, then, their output values $\mathbf{y}_{i}$ and $\mathbf{y}_{j}$ are highly correlated. 
The specific choice of the kernel function $k$ depends on the application, the most widely used being the squared exponential, or Gaussian, kernel

$$
k\left(\mathbf{x}, \mathbf{x}^{\prime}\right)=\sigma_{f}^{2} e^{-1 / 2\left(\mathbf{x}-\mathbf{x}^{\prime}\right) W\left(\mathbf{x}-\mathbf{x}^{\prime}\right)^{T}} .
$$

The kernel function is parameterized by $W$ and $\sigma_{f}$. The diagonal matrix $W$ defines the length scales of the process, which reflect the relative smoothness of the process along the different input dimensions and $\sigma_{f}^{2}$ is the signal variance.

In our application, we wish to learn a mapping from joint angles to muscle lengths. This is done by conditioning (9) on training data $D=\langle\mathbf{X}, \mathbf{Y}\rangle$, where $\mathbf{X}$ contains joint angles $\underline{\theta}$ and $\mathbf{Y}$ contains the corresponding muscle lengths $\underline{l}$ collected during the training phase. It can be shown that conditioning on training data and a test input $\mathbf{x}_{*}$ results in the following Gaussian predictive distribution over the output:

$$
p\left(\mathbf{y}_{*} \mid \mathbf{x}_{*}, D\right)=\mathcal{N}\left(\mathbf{y}_{*} ; \operatorname{GP}_{\mu}\left(\mathbf{x}_{*}, D\right), \operatorname{GP}_{\Sigma}\left(\mathbf{x}_{*}, D\right)\right)
$$

with mean

$$
\operatorname{GP}_{\mu}\left(\mathbf{x}_{*}, D\right)=\mathbf{k}_{*}^{T}\left[\mathbf{K}+\sigma_{\mathbf{n}}^{2} \mathbf{I}\right]^{-1} \mathbf{y}
$$

and variance

$$
\operatorname{GP}_{\Sigma}\left(\mathbf{x}_{*}, D\right)=k\left(\mathbf{x}_{*}, \mathbf{x}_{*}\right)-\mathbf{k}_{*}^{T}\left[\mathbf{K}+\sigma_{\mathbf{n}}^{2} \mathbf{I}\right]^{-1} \mathbf{k}_{*} .
$$

Here, $\mathbf{k}_{*}$ is a vector of kernel values between $\mathbf{x}_{*}$ and the training inputs $\mathbf{X}: \mathbf{k}_{*}[i]=k\left(\mathbf{x}_{*}, \mathbf{x}_{i}\right)$. Note that the prediction uncertainty, captured by the variance $\mathrm{GP}_{\Sigma}$, depends on both the process noise and the correlation between the test input and the training inputs. Here, we do not consider the prediction uncertainty, but focus on the GP mean prediction given by (12). As can be seen, the complexity of the GP mean prediction is linear in the number of training points. In our experiments, we found that GP prediction is efficient enough for finger control. If needed, however, more efficient predictions can be generated by sparsification of the GP [36].

The hyperparameters $\boldsymbol{\theta}_{y}$ of the GP are given by the parameters of the kernel function and the output noise: $\boldsymbol{\theta}_{y}=\left\langle\sigma_{n}, W, \sigma_{f}\right\rangle$. They can be determined by maximizing the log likelihood of the training data

$$
\boldsymbol{\theta}_{y}^{*}=\underset{\boldsymbol{\theta}_{y}}{\arg \max } \log p\left(\mathbf{Y} \mid \mathbf{X}, \boldsymbol{\theta}_{y}\right) .
$$

This optimization can be performed efficiently using techniques, such as conjugate gradient descent. Finally, the gradient of the GP mean-prediction function (12) gives the moment arm. For more details on GP, the reader is referred to [37].

\section{GP Example}

The following is a simple example of using GP for regression. This example demonstrates the ability of a GP to recover a function from noisy training data. In addition, the noise parameter of training data can also be recovered. The function we wish to learn is

$$
f(x, y)=\sin \left(\sqrt{x^{2}+y^{2}}\right) .
$$

This is shown in the first panel of Fig. 10 as a function of two inputs $x$ and $y$.

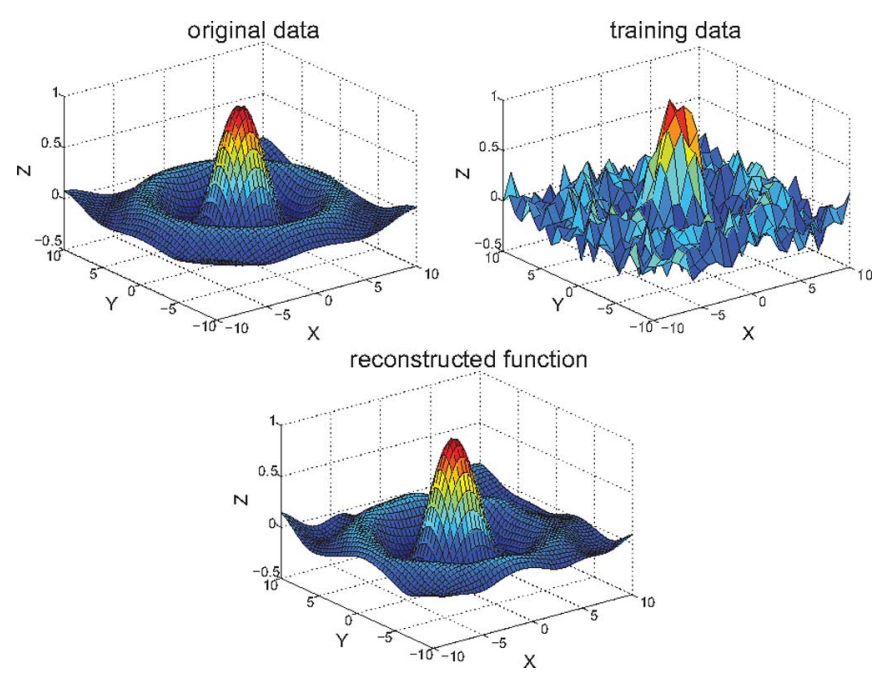

Fig. 10. (Left) Original function we wish to learn with a GP. (Middle) Noisy training data used for GP learning. (Right) Recovered function after GP optimization.

The training data consist of 625 noisy samples from this function at intervals of 0.8 on the grid, where $x$ and $y$ ranges from -10 to 10 . The noise value $\sigma$ used is 0.1 , i.e., at each point in the grid $[x, y]$, the training output is $z$ where

$$
z=f(x, y)+\mathcal{N}\left(0, \sigma^{2}\right) .
$$

The training data for the GP is $D=\langle(x, y), z\rangle$ and is displayed in the second panel of Fig. 10. Note the relatively high noise in the training data. The original function is greatly obscured by the noise.

After learning hyperparameters using conjugate gradient optimization, the GP can then be used to predict the the mean and variance of the recovered function, given a test input. We test using $x$ and $y$ at regularly spaced intervals of 0.4 along the grid. The resulting GP mean predictions are plotted in the last panel. One can see qualitatively how the recovered function closely matches the original function. Quantitatively, the rootmean-squared error for all the sample points is $1.3 \mathrm{e}-3$, where the function ranges from -0.5 to 1 . Another feature of the GP is the ability to learn the noise parameter of the training data. In this case, the mean prediction of the variance is 0.011 . This is very close to the true variance of 0.01 .

\section{REFERENCES}

[1] I. Albrecht, J. Haber, and H.-P. Seidel, "Construction and animation of anatomically based human hand models," in Proc. Eurograph./ SIGGRAPH Symp. Comput. Animation, 2003, pp. 98-109.

[2] K. N. An, Y. Ueba, E. Y. Chao, W. P. Cooney, and R. I. Linscheid, "Tendon excursion and moment arm of index finger muscles," J. Biomech., vol. 16, pp. 419-425, 1983.

[3] K.-N. An, "Tendon excursion and gliding: Clinical impacts from humble concepts," J. Biomech., vol. 40, pp. 713-718, 2007.

[4] R. Balasubramanian and Y. Matsuoka, "The role of small redundant actuators in precise manipulation," in Proc. IEEE/RSJ Int. Conf. Robot. Autom., 2009, pp. 4409-4415.

[5] Barrett. (2008). Barrett Technology Inc., Cambridge, MA. [Online]. Available: http://www.barrett.com/robot/index.htm

[6] D. J. Beevers and B. B Seedhom, "Metacarpophalangeal joint prostheses: A review of the clinical results of past and current designs," J. Hand Surg., vol. 20B, pp. 125-136, 1995. 
[7] A. Bicchi, "Hands for dexterous manipulation and robust grasping: A difficult road toward simplicity," IEEE Trans. Robot. Autom., vol. 16, no. 6, pp. 652-662, Dec. 2000.

[8] A. Bicchi and D. Prattichizzo, "Analysis and optimization of tendinous actuation for biomorphically designed robotic systems," Robotica, vol. 18, pp. 23-31, 2000.

[9] K. B. Biggers, S. C. Jacobsen, and G. E. Gerpheide, "Low level control of the UTAH/M. I. T. dexterous hand," presented at the IEEE Int. Conf. Robot. Autom., San Francisco, CA, 1986.

[10] P. W. Brand, K. C. Cranor, and J. C. Ellis, "Tendon and pulleys at the metacarpophalangeal joint of a finger," J. Bone Joint Surg., vol. 57, pp. 779-784, 1975.

[11] N. Brook, J. Mizrahi, M. Shoham, and J. Dayan, "A biomechanical model of index finger dynamics," Med. Eng. Phys., vol. 17, pp. 54-63, 1995

[12] W. Buford, S. Koh, C. R. Andersen, and S. F. Viegas, "Analysis of intrinsic-extrinsic muscle function through interactive 3-dimensional kinematic simulation and cadaver studies," J. Hand Surg., vol. 30, pp. 1267-1275, 2005

[13] M. C. Carrozza, P. Dario, F. Vecchi, S. Roccella, M. Zecca, and F. Sebastiani, "The CyberHand: On the design of cybernetic prosthetic hand intended to be interfaced to the peripheral nervous system," in Proc. IEEE/RSJ Int. Conf. Intell. Robots Syst., 2003, pp. 2642-2647.

[14] A. D. Deshpande, R. Balasubramanian, R. Lin, B. Dellon, and Y. Matsuoka, "Understanding variable moment arms for the index finger MCP joints through the ACT hand," in Proc. IEEE BIOROB, 2008, pp. 776-782.

[15] A. D. Deshpande, J. Ko, D. Fox, and Y. Matsuoka, "ACT hand finger control: Muscle and joint torque control strategies," in Proc. IEEE Int. Conf. Robot. Autom., 2009, pp. 4416-4422.

[16] Y. Engel, P. Szabo, and D. Volkinshtein, "Learning to control an octopus arm with gaussian process temporal difference methods," in Advances in Neural Information Processing Systems. Cambridge, MA: MIT Press, 2006, pp. 347-354.

[17] A. G. Feldman, "Functional tuning of the nervous system with control of movement or maintenance of a steady posture-II. Controllable parameters of the muscles," Biophysics, vol. 11, pp. 565-578, 1966.

[18] N. Fowler, A. Nicol, B. Condon, and D. Hadley, "Method of determination of three dimensional index finger moment arms and tendon lines of action using high resolution mri scans," J. Biomech., vol. 34, pp. 791-797, 2001.

[19] J. L. Fu and N. S. Pollard, "On the importance of asymmetries in grasp quality metrics for tendon driven hands," in Proc. IEEE/RSJ Int. Conf. Intell. Robots Syst., 2006, pp. 1068-1075.

[20] K. R. S. Holzbaur, W. M. Murray, and S. L. Delp, "A model of the upper extremity for simulating musculoskeletal surgery and analyzing neuromuscular control," Ann. Biomed. Eng., vol. 33, pp. 829-840, 2005

[21] iLimb: Touch Bionics. (2010, Jan. 8). Ilimb. [Online]. Available: http://www.touchbionics.com/i-LIMB

[22] S. C. Jacobsen, D. F. Iversen, R. T. Knutti, and K. B. Biggers, "Design of the UTAH/M. I. T. dexterous hand," in Proc. IEEE Int. Conf. Robot. Autom., 1986, pp. 1520-1532.

[23] D. G. Kamper, H. C. Fischer, and E. G. Cruz, "Impact of finger posture on mapping from muscle activation to joint torque," Clin. Biomech., vol. 21, pp. 361-369, 2006

[24] M. Kawato, K. Furuwaka, and R. Suzuki, "A hierarchical neural network model for the control and learning of voluntary movements," Biol. Cybenetics, vol. 57, pp. 169-185, 1987.

[25] J. Ko and D. Fox, "Gp-bayesfilters: Bayesian filtering using gaussian process prediction and observation models," in Proc. IEEE/RSJ Int. Conf. Intell. Robots Syst., 2008, pp. 3471-3476.

[26] J. Ko, D. Klein, D. Fox, and D. Hähnel, "Gaussian processes and reinforcement learning for identification and control of an autonomous blimp," in Proc. IEEE Int. Conf. Robot. Autom., 2007, pp. 742-747.

[27] D. Kuechle, S. Newman, E. Itoi, B. Morrey, and K. An, "Shoulder muscle moment arms during horizontal flexion and elevation," J. Shoulder Elbow Surg., vol. 6, pp. 429-439, 1997.

[28] K.-H. Lee and K. H. Kroemer, "A finger model with constant tendon moment arms," in Proc. Hum. Fact. Ergonom. Soc. 37th Annu. Meeting, 1993, pp. 710-714.

[29] Z. M. Li, V. M. Zatsiorsky, and M. L. Latash, "Contribution of the extrinsic and intrinsic hand muscles to the moments in finger joints," Clin. Biomech., vol. 15, pp. 203-211, 2000.

[30] Z.-M. Li, H. J. Pfaeffle, D. G. Sotereanos, R. J. Goitz, and S. L.-Y. Woo, "Multi-directional strength and force envelope of the index finger," Clin. Biomech., vol. 18, pp. 908-915, 2003.
[31] S. Narasimhan, D. M. Siegel, and J. M. Hollerbach, "Condor: An architecture for controlling the utah-mit dexterous hand," IEEE Trans. Robot. Autom., vol. RA-5, no. 5, pp. 616-627, Oct. 1989.

[32] U. D. D. S. Office. (2010, Jan. 8). Revolutionizing prosthetics [Online]. Available: http://www.darpa.mil/dso/thrusts/bio/restbio_tech/revprost/ index.htm

[33] N. S. Pollard, "Closure and quality equivalence for efficient synthesis of grasps from examples," Int. J. Robot. Res., vol. 23, pp. 595-613, 2004.

[34] N. S. Pollard and R. C. Gilbert, "Tendon arrangement and muscle force requirements for human like force capabilities in a robotic finger," in Proc. IEEE Int. Conf. Robot. Autom., 2002, pp. 3755-3762.

[35] B. M. Quaney, R. J. Nudo, and K. J. Cole, "Can internal models of objects be utilized for different prehension tasks?" J. Neurophysiol., vol. 93, pp. 2021-2027, 2005.

[36] J. Quinonero Candela and C. Rasmussen, "A unifying view of sparse approximate Gaussian process regression," J. Mach. Learning, vol. 6 , pp. 1939-1959, 2005.

[37] C. Rasmussen and C. Williams, Gaussian Processes for Machine Learning. Cambridge, MA: MIT Press, 2005.

[38] RTAI. [Online]. Available: http://www.rtai.org

[39] S. Sueda, A. Kaufman, and D. K. Pai, "Musculotendon simulation for hand animation," in Proc. ACM SIGGRAPH, vol. 27, no. 3, Aug. 2008

[40] A. B. Swanson, "Silicone rubber implants for replacement of arthritic or destroyed joints in the hand," Surg. Clin. North Amer., vol. 48, pp. 1113$1127,1968$.

[41] M. V. Weghe, M. Rogers, M. Weissert, and Y. Matsuoka, "The ACT hand: Design of the skeletal structure," in Proc. IEEE Int. Conf. Robot. Autom., 2004, pp. 3375-3379.

[42] A.-P. C. Weiss, D. C. Moore, C. Infantolino, J. J. Crisco, E. Akelman, and R. D. McGovern, "Metacarpophalangeal joint mechanics after 3 differen silicone arthroplasties," J. Hand Surg., vol. 29A, pp. 796-803, 2004

[43] D. D. Wilkinson, M. V. Weghe, and Y. Matsuoka, "An extensor mechanism for an anatomical robotic hand," in Proc. IEEE Int. Conf. Robot. Autom. 2003, pp. 238-243.

[44] D. M. Wolpert and Z. Ghahramani, "Computational principles of movement neuroscience," Nature Neurosci., vol. 3, pp. 1212-1217, 2000.

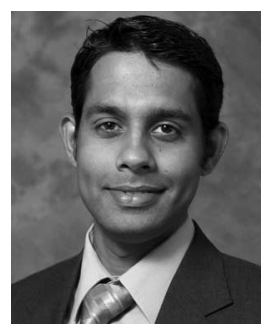

Ashish D. Deshpande received the Ph.D. degree in mechanical engineering from the University of Michigan, Ann Arbor, in 2007.

He was a Research Associate in the NeuRobotics Laboratory, University of Washington, Seattle. He is currently an Assistant Professor in the Department of Mechanical Engineering, University of Maine, Orono, ME. His research interests include development of new methodologies to represent full dynamics of general robotic systems, design and development of search and rescue robot teams, implementation of robotics methods for human motion modeling and design of prostheses, development of robot devices based on biomechanical analyses to assist in rehabilitation and to improve prostheses design, study of neuromuscular controls in humans to implement human-like motion and force controls in anatomical robotic systems, and study of the dynamics and controls of multibody robotic systems and their application to human-motion analysis.

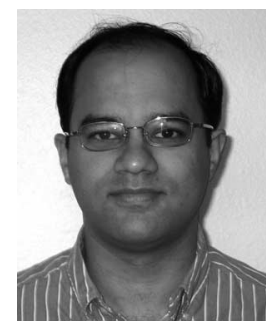

Ravi Balasubramanian received the B.Eng. degree and M.Eng. degrees in mechanical engineering from the National University of Singapore, Singapore, in 1999 and 2000, respectively, and the Ph.D. degree in robotics from Carnegie Mellon University, Pittsburgh, PA, in 2006.

Between 2006 and 2009, he was a Postdoctoral Research Associate in neurobotics at the University of Washington, Seattle. He is currently an Associate Research Scientist in mechanical engineering at Yale University, New Haven, CT. His research interests include robotics and human control systems.

Dr. Balasubramanian was the Best Student Paper Finalist at the IEEE International Conference on Robotics and Automation for his doctoral work on a novel locomotion technique called legless locomotion in 2004. 


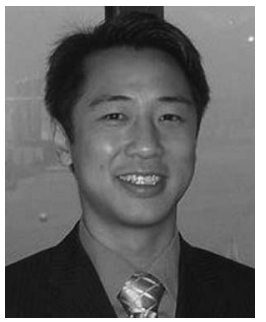

Jonathan Ko is currently working toward the Ph.D degree from the Department of Computer Science, University of Washington, Seattle.

His current research interests include machine learning for robotic systems, in particular, Gaussian processes for modeling of dynamical systems.

Mr. Ko was a recipient of the Sarcos Best Student Paper Award in the Conference of Intelligent Robots and Systems 2007 for his paper titled "GP-UKF: Unscented Kalman Filters with Gaussian Process Prediction and Observation Models."

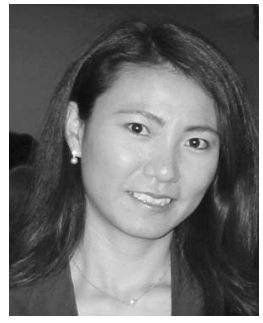

Yoky Matsuoka received the B.S. degree in electrical engineering and computer science from the University of California, Berkeley, in 1993, and the M.S. degree in electrical engineering and computer science and $\mathrm{Ph} . \mathrm{D}$. degree in electrical engineering and computer science in the fields of artificial intelligence and computational neuroscience from Massachusetts Institute of Technology, Cambridge, in 1995 and 1998, respectively.

She is currently the Torode Family Endowed ProUniversity of Washington, Seattle.

fessor in computer science and engineering at the

Dr. Matsuoka was the Anna Loomis McCandless Chair in 2004. She was the MacArthur Foundation Fellow in 2007. She was a recipient of the Presidential Early Career Award for Scientists and Engineers in 2004 and the IEEE Robotics and Automation Society Early Academic Career Award in 2005. 